\title{
Autoeficacia e intención conductual del consumo de tabaco en adolescentes: validez factorial y relación estructural
}

\section{Smoking-related self-efficacy and smoking intention in young people: Factorial validity and structural association.}

\author{
| Jorge Raúl Palacios Delgado
}

| Facultad de Psicología. Universidad Nacional Autónoma de México

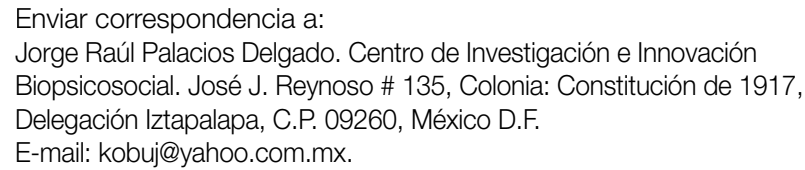

\section{RESUMEN}

El objetivo de la presente investigación fue obtener en un modelo de ecuaciones estructurales, la validez factorial de la autoeficacia ante el consumo de tabaco, asi como su relación estructural con la conducta e intención tabáquica. Se seleccionó una muestra de 1012 jóvenes entre 14 y 22 años de la Ciudad de México. La conducta de fumar se midió considerando la edad de inicio del consumo de tabaco, la frecuencia y la cantidad de consumo. Además se preguntó la probabilidad de fumar cigarros en un futuro. La autoeficacia se evaluó con ítems que reflejan la capacidad para evitar consumir cigarros. Los resultados mostraron validez factorial de la medida de autoeficacia en esta muestra. El análisis de modelo de ecuaciones estructurales $\left(X^{2}=113.319, p<.001\right.$; $\mathrm{CFI}=.99 ; \mathrm{NFI}=.99 ; \mathrm{GFI}=.95 ; \mathrm{RMSEA}=.05)$ sugiere que los jóvenes que tienen una menor capacidad para evitar consumir tabaco tendrán una mayor intención y conducta de fumar, además la intención futura de fumar predice su consumo. La discusión analiza la utilidad de los resultados para la evaluación de la autoeficacia en adolescentes, así como las implicaciones clínicas para las personas que se encuentran en tratamiento.

Palabras clave: Consumo de tabaco, autoeficacia, intención conductual, jóvenes y ecuaciones estructurales.

\section{ABSTRACT}

The objective of this research was to obtain, in a structural equation model, the factorial validity of smoking-related self-efficacy, as well as its structural association with behavior and smoking intention. The sample was made up of 1012 young people aged 14 to 22 (mean 16.76) from public high schools in Mexico City. These young people filled out a questionnaire that assessed tobacco use (current smokers' age at first cigarette, smoking frequency and quantity of cigarettes smoked). They were also asked about intention to smoke in the future. Self-efficacy was assessed using items that measured one's capacity to avoid smoking. The results indicated the factorial validity of the smoking-related self-efficacy measure in this sample. Structural equation modeling analyses suggested $\left(X^{2}=113.319, \mathrm{p}<.001 ; \mathrm{CFI}=.99 ; \mathrm{NFI}=.99 ; \mathrm{GFI}=.95 ; \mathrm{RMSEA}=.05\right)$ that those young people with lower confidence in their ability to avoid smoking would have higher levels of smoking intention and smoking behavior; moreover, intention to smoke in the future predicts level of smoking. The discussion analyzes the utility of the results for the assessment of selfefficacy, as well as the clinical implications for the people in treatment.

Key Words: Tobacco smoking, self-efficacy, smoking intention, young people, structural equation modeling. 
E consumo de sustancias adictivas es un comportamiento que adquiere especial importancia en la adolescencia y la juventud (Ortiz, Soriano, Galvan y Meza, 2005). El Instituto Nacional de Estadística, Geografía e Informática (INEGI) en el año 2005 señaló que el tabaquismo se asocia estrechamente con los principales indicadores de morbilidad y mortalidad en México, por lo que constituye un importante problema de salud pública. Se estima que alrededor de 122 personas mueren diariamente en México por causas asociadas con el tabaco. Asimismo, las enfermedades cardiovasculares, broncopulmonares y diversos tipos de cáncer entre los que se encuentra el cáncer de pulmón, constituyen las principales causas de mortalidad.

En nuestro país, en el 2002 la Encuesta Nacional de Adicciones (ENA) señaló que la prevalencia de fumadores entre los adolescentes de 12 a 17 años de zonas urbanas equivale a casi un millón de individuos, con una mayor proporción de hombres (15.4\%) que de mujeres (4.8\%), es decir, 3 varones por cada mujer.

Por otra parte, la investigación señala que existen una serie de teorias y modelos que se utilizan para explicar el consumo de tabaco por la complejidad que tiene este comportamiento y su naturaleza multicausal, dentro de estas explicaciones se encuentran la Teoría de Acción Razonada (Fishbein, 1990) y de la Conducta Planeada (Ajzen, 1991), el Modelo Transteórico del cambio (Prochaska y DiClemente, 1983), la Teoría del la Influencia Triádica (Flay y Petraitis, 1994) y la Teoría Sociocognitiva de Bandura $(1977,1987)$.

La Teoría Social Cognitiva desarrollada por Bandura (1977, 1987, 1997) es una de las teorías más utilizadas dentro del campo de las conductas adictivas, y un elemento importante dentro de esta teoría es el constructo de autoeficacia, de los cuales distingue dos tipos de expectativas, las expectativas de resultado y las expectativas de eficacia (autoeficacia). La autoeficacia se define como la capacidad o convicción personal que tiene el sujeto de poder ejecutar exitosamente la conducta requerida para actuar o producir un resultado en una situación, actividad o dominio (Bandura, 1997).

La autoeficacia tiene como elemento central las percepciones que tienen los individuos de su capacidad de actuar. Desde la perspectiva de la teoría social cognitiva, la autoeficacia percibida afecta los propios niveles de motivación y ejecución del individuo, a través de su capacidad para afrontar los cambios. Un postulado importante de la teoría de la autoeficacia $(1977,1997)$ señala que las percepciones de eficacia varian de modo significativo entre las distintas situaciones y tareas, de esta manera la autoeficacia se centra en los pensamientos que los individuos tienen acerca de su capacidad para actuar.

Cuando se habla de autoeficacia se hace en un sentido amplio, es decir, se hace referencia a la capacidad para manejar adecuadamente una amplia gama de situaciones de la vida cotidiana y por lo regular su medición se realiza de forma general (Pérez, Bermúdez y Sanjuán, 2000), también la autoeficacia se ha medido a través de su relación con conductas en específico para determinar de manera más precisa su influencia en el dominio de interés. Sin embargo, a pesar de que se cuenta con medidas de autoeficacia para evitar conductas adictivas (Dijkstra y De Vries, 2000; Fagan, Eisenberg, Frazier, Stoddard, Avrunin, y Sorensen, 2003; Gwaltney, Shiffman, Norman, Paty, Kassel, Gnys, Hickcox y Balanbis, 2001; Martín, Wilkinson y Poulos, 1995) en pocas ocasiones se muestran las propiedades psicométricas de estas escalas en adolescentes. Además la evidencia de validez factorial para medir autoeficacia ante el consumo de tabaco o su intención, la mayoría de las veces se hace con personas que han intentado dejar de fumar o en población adulta. Adicionalmente los ítems de las escalas que intentan operacionalizar la autoeficacia relacionada con el consumo de tabaco lo realizan en situaciones concretas o con variables situacionales especificas.

De acuerdo con los resultados obtenidos con los diferentes autores, considero que la medición de la autoeficacia relacionada con el consumo de tabaco implica contextos, situaciones y conductas especificas, es decir, una conducta (fumar) se puede presentar de una forma particular, por ejemplo, con los amigos, en la escuela, en una fiesta, etc., de manera que los cambios asociados con esta variedad de situaciones, sugiere en el adolescente la habilidad y capacidad necesaria para evitar involucrarse en conductas que ponen en riesgo su salud en varias situaciones o contextos en el cual se encuentra constantemente.

Por otra parte, los mecanismos por los cuales la autoeficacia influye a la intención y la conducta de fumar en adolescentes fumadores ha sido poco estudiada. Algunos estudios (Calleja, 1998; Carvajal y Granillo, 2006; Carvajal, Hanson, Downing, Coyle y Pederson, 2004; Flay et al, 1994) han demostrado que las dimensiones cognoscitivas afectan una determinada conducta y la adopción de un curso de acción en particular. Especificamente se ha encontrado que la autoeficacia es un determinante de la conducta, ya que a partir de la misma es posible predecir la conducta, en este sentido la autoeficacia se ha puesto a prueba para predecir el consumo de sustancias adictivas. Por ejemplo, si un adolescente no se siente capaz de resistir la tentación de consumir tabaco (baja autoeficacia percibida), tendrá mayor probabilidad de fumar o de actuar bajo la influencia social (Carvajal et al, 2004; Carvajal et al, 2006). La autoeficacia percibida afecta el consumo de substancias adictivas, las personas con alta autoeficacia son más resistentes al consumo de substancias adictivas que quienes no tienen un fuerte control personal. La experiencia que se tenga con el consumo de tabaco afecta el nivel de eficacia, de manera que un menor nivel de autoeficacia se relaciona con el consumo de tabaco. Por lo tanto, la baja eficacia percibida para evitar el consumo contribuye en la predicción del abuso de substancias (Bandura, 1999; Schonfield, Pattison, Hill y Borland, 2003).

Fagan, Eisenberg, Frazier, Stoddard, Avrunin y Sorensen (2003) encontraron que quienes fuman a diario tuvieron menos autoeficacia para evitar fumar, en comparación con los que fuman con menos frecuencia. Los adolescentes que han intentado fumar tuvieron una menor puntuación en autoeficacia que quienes no intentaron fumar. Para Landrum, 
Diamond, Dolan, Pallonen, Ford y McAlister (2007) la autoeficacia para evitar fumar tiene una influencia pequeña pero no significativa en la intención de fumar. Considerado los resultados obtenidos, los autores realizaron modificaciones al modelo conseguido, obteniendo que la autoeficacia tiene un efecto indirecto sobre la intención de fumar a través de creencias relacionadas con el consumo.

La intención de fumar se ha identificado como un predictor del consumo en jóvenes (Calleja y Aguilar, 2008; Carvajal et al, 2004; Carvajal et al, 2006; Schonfield et al, 2003), sin embargo, pocos estudios han demostrado la dirección y magnitud a través del cual constructos cómo la autoeficacia relacionada con el consumo de tabaco inciden en la intención conductual para fumar y cómo ambos influyen en la consumo de tabaco en jóvenes.

Considerando lo antes planteado, el modelo conceptual que se pretende estimar se encuentra derivado de una combinación de constructos de teorías de cambio conductual (Fishbein, 2000) y de la teoria social cognitiva (Bandura 1977, 1987). El modelo plantea que la conducta de fumar probablemente se presente si no se cuenta con las capacidades necesarias para evitar o rechazar la conducta. La intención conductual de fumar esta influida por la eficacia con respecto a la conducta. El modelo hipotetiza que la autoeficacia relacionada con el tabaco tiene un efecto directo sobre la intención y conducta de fumar e indirecto a través de la intención conductual para fumar.

Evaluar el modelo planteado en adolescentes que fuman puede otorgar evidencia de cómo aspectos cognitivo-conductuales pueden ser utilizados tanto en la prevención como en el pronostico de consumo en los jóvenes. Derivado del planteamiento presentado hasta el momento, el objetivo de la presente investigación fue obtener en un modelo de ecuaciones estructurales, la validez factorial de la autoeficacia ante el consumo de tabaco, así como su relación estructural con la conducta e intención tabáquica en una muestra de adolescentes mexicanos.

\section{MÉTODO}

\section{Participantes}

Se seleccionó una muestra no probabilística de 1012 jóvenes, 531 hombres y 481 mujeres, con un rango de edad entre 14 y 22 años y una media de 16.75 (DE = 1.2) años. Participaron 326 jóvenes del primer año, 246 del segundo año y 440 del tercer año, estudiantes de dos escuelas de educación media superior del Distrito Federal de la Ciudad de México, de ambos turnos, 528 del turno matutino y 484 del vespertino. Su participación fue voluntaria y se les garantizó el anonimato en sus respuestas.

\section{Instrumentos}

La conducta de fumar se midió considerando la edad de inicio del consumo de tabaco, la frecuencia y la cantidad de consumo en el último año. Para medir la intención conduc- tual de fumar, se les preguntó la probabilidad de fumar cigarros en los próximos 6 meses.

Para medir la autoeficacia relacionada con el consumo de tabaco se desarrolló un instrumento específico que mide la autoeficacia para evitar o rechazar consumir cigarros partiendo de ítems similares de otras escalas que miden autoeficacia para evitar fumar (Dijkstra et al, 2000; Fagan et al 2003; Gwaltney et al, 2001), así como la redacción de ítems ex profeso para esta investigación. Esta nueva medida indica el nivel de confianza o capacidad que los adolescentes tienen para evitar fumar (consumir tabaco) en diferentes lugares, contextos, actividades o situaciones en los cuales se consume tabaco regularmente, así como ítems que reflejan la presencia de amigos que consumen tabaco sin que el propio adolescente lleve acabo la conducta, utilizando acciones dirigidas para negarse a consumir (p.ej., Me siento capaz de negarme a fumar cigarros si me los ofrecen). Es una escala tipo Likert con un rango de respuestas de cuatro niveles que van de nunca a siempre. Las respuestas pueden ser sumadas para obtener un índice de autoeficacia, el resultado de la puntuación (altos valores) indica una elevada autoeficacia para evitar consumir tabaco.

\section{RESULTADOS}

De los 1012 adolescentes que participaron en este estudio, el $68.3 \%$ ha consumido tabaco alguna vez en la vida, de éstos el 17\% fuman una vez al mes o menos, el 18.7\% solo lo ha hecho una vez y el $14.4 \%$ fuma diario o casi diario, con un promedio de 2 cigarros $(D E=2 ;$ rango $=1$ a 25$)$ diarios. $\mathrm{La}$ edad promedio de inicio del consumo es a los $14(D E=1.9$; rango $=5$ a 20) años de edad para hombres y mujeres.

No se encontraron diferencias en el consumo de tabaco entre hombres y mujeres (frecuencia de consumo [hombres $\mathrm{M}=3.29, \mathrm{DE}=2.24 ;$ mujeres $\mathrm{M}=3.06, \mathrm{DE}=2.1 ;$ rango $=1 \mathrm{a}$ $7 ; \mathrm{t}=1.61, p>.05]$, cantidad de cigarros [hombres $\mathrm{M}=2.23$, $\mathrm{DE}=2.5$; mujeres $\mathrm{M}=1.92, \mathrm{DE}=1.8$; rango $=1$ a $25 ; \mathrm{t}=1.86$, $p>.05$ ], edad de inicio [hombres $\mathrm{M}=14, \mathrm{DE}=2$; mujeres $\mathrm{M}=$ $14, \mathrm{DE}=2 ;$ rango $=5$ a $20 ; \mathrm{t}=.14, p>.05])$. Con el propósito de obtener la equivalencia métrica de los indicadores del consumo de tabaco (frecuencia, cantidad y edad de inicio), cada indicador se transformo a puntuaciones estandarizadas para obtener su distribución normal y así poderlos sumar en un indicador global de consumo de tabaco.

Por otra parte, se realizó un análisis factorial confirmatorio (AFC) para establecer la validez factorial de la medida de autoeficacia relacionada al tabaco en esta muestra (Tabla 1). Los análisis realizados con el AFC en los adolescentes consumidores de tabaco mostró que sin ser necesariamente el óptimo, éste se ajusta a los datos, proporcionando validez factorial a la medida desarrollada. Los indicadores de bondad de ajuste absoluto $\left[X^{2}(5, N=1012)=66.05, p<.001 ; G F I=\right.$ $.97 ; \mathrm{RMSEA}=.08 ; p-$ Close $=0.00]$; de ajuste incremental $(\mathrm{TLI}=.95 ; \mathrm{RFI}=.94 ; \mathrm{IFI}=.97, \mathrm{CFI}=.97)$ y de ajuste de parsimonia $(\mathrm{AGFI}=.92, \mathrm{NFI}=.97)$ mostraron ser adecuados. 
Tabla 1. Carga factorial, media, desviación estándar y varianza explicada de los ítems de la medida de autoeficacia ante el consumo de tabaco.

\begin{tabular}{|c|c|c|c|c|}
\hline İtems & Media & $\begin{array}{c}\text { Desviación } \\
\text { Estándar }\end{array}$ & Carga Factorial & R2 \\
\hline R24 & 2.93 & 1.1 & .71 & .51 \\
\hline R151 & 3.28 & 1.0 & .74 & .55 \\
\hline R28 & 2.97 & 1.1 & .74 & .55 \\
\hline R75 & 3.15 & 1.1 & .83 & .69 \\
\hline R59 & 3.03 & 1.1 & .79 & .63 \\
\hline
\end{tabular}

Para obtener la fiabilidad de los ítems se calculó el índice de consistencia interna (coeficiente alpha de Cronbach) para el total del instrumento, obteniendo un índice de .92 (IC95\% $=.91-.92$ ). Los resultados sugieren niveles de fiabilidad satisfactorios, lo que indica una homogeneidad en los items que componen la medida de autoeficacia.

Un modelo de ecuaciones estructurales fue especificado para estimar empíricamente el modelo conceptual planteado en la presente investigación entre la autoeficacia y la intención y conducta de fumar. El modelo hipotetizado fue estructuralmente consistente con los datos. El análisis del modelamiento estructural de ecuaciones sugiere que la autoeficacia relacionada con el consumo de tabaco tiene un efecto directo (coeficiente estructural de -.65) sobre la intención y conducta de fumar (coeficiente estructural de -.40). El modelo también sugiere que la autoeficacia tiene un efecto indirecto hacia la conducta a través de la intención (coeficiente estructural de .49), es decir, los jóvenes que tienen una menor capacidad para evitar consumir tabaco tendrán una mayor intención y conducta de fumar, además la intención futura de fumar predice el consumo en los adolescentes.

El modelo presenta un coeficiente de determinación múltiple $\mathrm{R}^{2}$ en la estimación de la conducta de fumar de .66, lo que significa que en este modelo, la autoeficacia relacionada con el consumo de tabaco y la intención conductual de fumar explican el $66 \%$ de la variabilidad del consumo de tabaco en jóvenes mexicanos. Además la intención conductual se encuentra explicada en un $41 \%$ de la varianza por la autoeficacia para rechazar el consumo de cigarros.

Los indicadores de bondad de ajuste absoluto, $\left[X^{2}(25, N=\right.$ $1012)=113.319, \mathrm{p}<.001 ; X^{2} / \mathrm{gl}=4.53 ; \mathrm{GFI}=.95 ; \mathrm{RMSEA}=$ $.05 ; \mathrm{p}-$ Close $=0.08] ;$ de ajuste incremental $(\mathrm{TLI}=.99 ; \mathrm{RFI}=$ $.98 ; \mathrm{IFI}=.99, \mathrm{CFI}=.99)$ y de ajuste de parsimonia $(\mathrm{AGFI}=.94$, $\mathrm{NFI}=.99$ ) fueron favorables, lo que permite afirmar que el modelo teórico postulado y sus relaciones está respaldado por los datos, con un ajuste adecuado a los mismos. La Figura 1 muestra los resultados y los parámetros estandarizados obtenidos del modelo estructural. Las abreviaturas utilizadas en el modelo se refieren a la Frecuencia $=$ Frec, la Cantidad $=$ Cant, y la Edad de inicio en el consumo de tabaco: Edad.

\section{DISCUSIÓN}

Los resultados obtenidos respecto a la prevalencia del consumo de tabaco es similar con la ENA (2002), la diferencia estadística mostró que hombres y mujeres consumen la misma cantidad de tabaco. El hecho de que hombres y mujeres fumen de igual forma indica que en años futuros los jóvenes de ambos sexos presentarán problemas de salud, duplicando el costo para las instituciones de salud que tengan que cubrir los daños ocasionados por el cigarro. Al analizar la edad promedio del inicio del consumo de tabaco, así

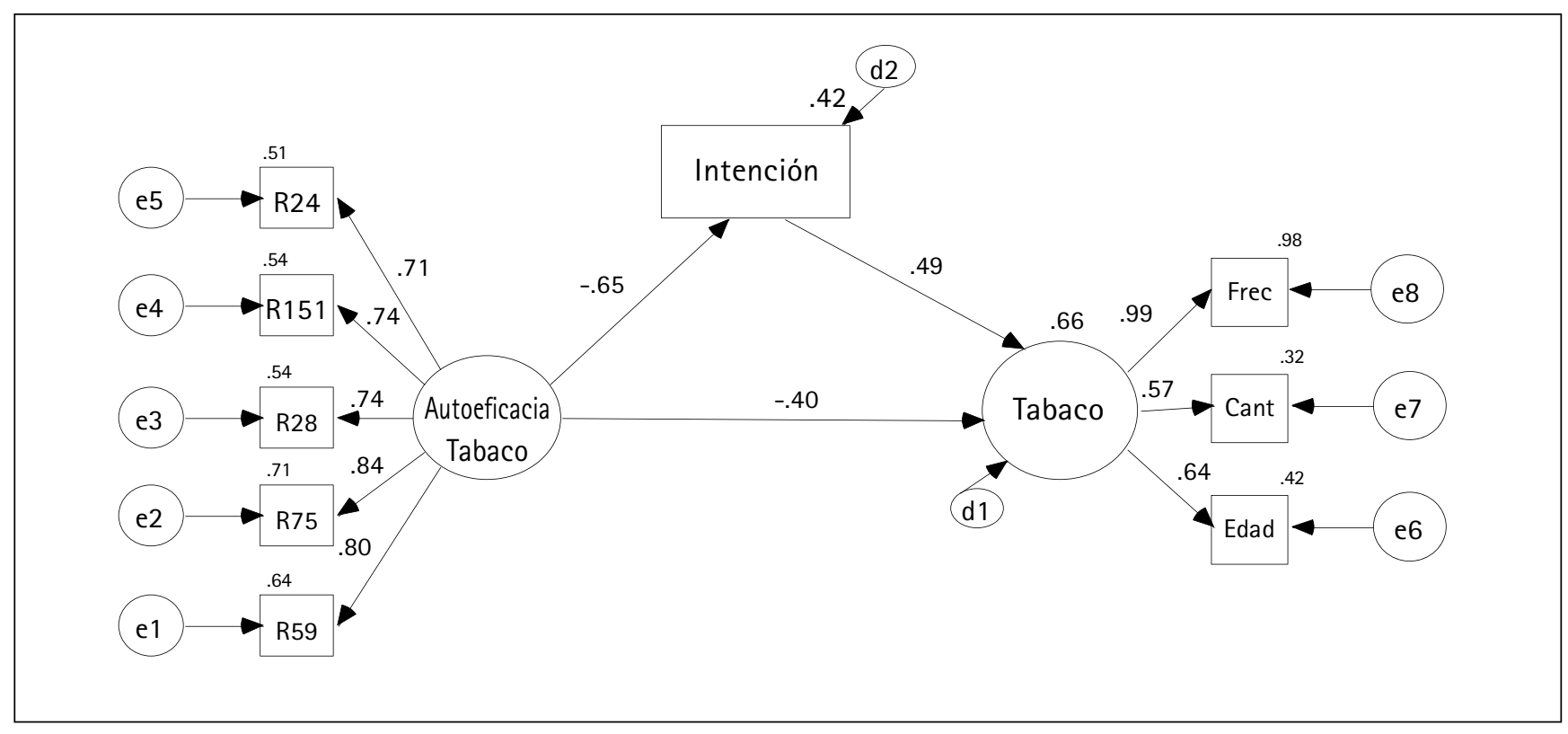

Figura 1. Estimación del modelo estructural de la autoeficacia e intención conductual del consumo de tabaco en adolescentes. 
como el valor observado por la desviación estándar, muestra que éste se llevó a cabo mientras los adolescentes cursaban la enseñanza secundaria; por lo que este nivel educativo se considera un sector propicio para la prevención de la conducta tabáquica, además de que los últimos años de enseñanza primaria, resultan ser poblaciones idóneas para iniciar los programas de prevención, así como, para promover una educación para la salud.

La evidencia encontrada provee apoyo a la validez factorial de la autoeficacia relacionada al consumo de tabaco. Consistente con la teoría social cognitiva y con la autoeficacia (Bandura, 1977, 1987, 1997), los resultados obtenidos indican que los adolescentes pueden evitar involucrarse en conductas de riesgo a través de diferentes situaciones o contextos, medidos por medio de cinco ítems. Los ítems fueron desarrollados para medir la autoeficacia ante el consumo de tabaco y estuvieron diseñados para incluir una serie de situaciones o contextos bajo los cuales los adolescentes se pueden encontrar cuando se realizan estos comportamientos.

La evaluación factorial obtenida en este estudio refleja el contenido para medir autoeficacia relacionado al consumo de tabaco (Dijkstra et al, 2000; Fagan et al 2003; Gwaltney et al, 2001) pero a través de varios contextos o situaciones. La evidencia de las propiedades psicométricas de validez y fiabilidad, muestra congruencia conceptual entre los ítems, reafirmando la validez factorial de los ítems tal y como han sido evaluados en el presente estudio.

Los hallazgos encontrados en el modelo estructural sugieren que la autoeficacia ante el consumo de tabaco tiene un efecto directo sobre la conducta e intención de fumar, contrario a los resultados obtenidos por Landrum et al (2007) quienes señalan un efecto indirecto mediado por las creencias de fumar. Lo encontrado en ésta investigación está apoyado teórica (Ajzen, 1991; Bandura 1977, 1983, 1997; Fishbein, 1990; Fishbein, 2000) y empíricamente (Calleja et al, 2008; Carvajal et al, 2006; Fagan et al, 2003) de manera que el efecto de la autoeficacia sobre la conducta e intención de fumar apoya lo encontrado por otros estudios (Carvajal et al, 2004; Carvajal et al, 2006; Fagan et al, 2003; Schonfield et al, 2003).

La aportación de este estudio fue obtener la validez factorial de ítems para medir la autoeficacia ante el consumo de tabaco de una manera válida, fiable y culturalmente sensible para medir este tipo de autoeficacia en adolescentes mexicanos al incorporar situaciones o contextos específicos bajo los cuales se pueden realizar estas conductas. Una segunda aportación fue estimar empíricamente el modelo planteado con la finalidad de conocer la dirección y magnitud que tiene la autoeficacia sobre la conducta e intención de fumar cigarros. De estos resultados se derivan implicaciones clínicas debido a que la escala obtenida puede ser útil para la elaboración y evaluación de personas que consumen tabaco o quienes se encuentran bajo tratamiento para dejar de consumir alguna sustancia adictiva, ya que se observa que los consumidores de tabaco tienen menos confianza en su habilidad para resistir o abstenerse de consumir sustancias adictivas (Gwaltney et al, 2001).
Las consecuencias del consumo de sustancias abarcan desde ámbitos individuales, sociales y sanitarios como familiares y económicos, etc. Ante este hecho uno de los modos que parece idóneo para abordar esta problemática es mediante la prevención del consumo de sustancias adictivas (Becoña, 2006). Los programas realizados para tal fin deberán contemplar aspectos cognitivo-conductuales como los planteados en este estudio, si se pretende obtener efectos positivos a través de las intervenciones realizadas a lo largo del tiempo.

Por último se debe tener en cuenta que estos resultados no se pueden generalizar a toda la población adolescente, sino que reflejan lo que está pasando con una muestra de jóvenes de educación media superior y que para corroborar los resultados obtenidos se debería replicar el estudio en otras muestras.

\section{REFERENCIAS}

Ajzen, I. (1991). The theory of planned behavior. Organizational Behavior and Human Decisions Processes, 50, 179-211.

Bandura, A. (1977). Self-efficacy: Toward a unifying theory of behavioral change. Psychological Review, 84, 191- 215.

Bandura, A. (1987). Pensamiento y acción: fundamentos sociales. Barcelona: Martínez Roca.

Bandura, A. (1997). Self-efficacy: the exercise of control. New York: W.H. Freeman.

Bandura, A. (1999). A sociocognitive analysis of substance abuse: An agentic perspective. Psychological Science, 10, 214- 217.

Becoña, E. (2006). Tabaco. Prevención y tratamiento. Madrid: Pirámide.

Calleja, B. N. (1998). Creencias sobre las consecuencias del tabaco. La Psicologia Social en México, 7, 254- 259.

Calleja, B. N. y Aguilar, J. (2008). Por qué fuman las adolescentes: Un modelo estructural de la intención de fumar. Adicciones, 20, 387- 394.

Carvajal, S. C. y Granillo, T. M. (2006). A prospective test of distal and proximal determinants of smoking initiation in early adolescents. Addictive Behaviors, 31, 649- 660.

Carvajal, S. C., Hanson, C., Downing, R. A., Coyle, K. y Pederson, L. (2004). Theory-based determinants of youth smoking: A multiple influence approach. Journal of Applied Social Psychology, 34, 59- 84.

Dijkstra, A. y De Vries, H. (2000). Clusters of precontemplating smokers defined by the perceptions of the pros, cons and self-efficacy. Addictive Behaviors, 25, 373- 385.

Encuesta Nacional de Adicciones (ENA) (2002). Tabaco. Secretaria de Salud, Consejo Nacional contra las Adicciones, Instituto Nacional de Psiquiatría, Dirección General de Epidemiología, Instituto Nacional de Estadistica, Geografía e Informática.

Fagan, P., Eisenberg, M., Frazier, L., Stoddard, A. M. Avrunin, J. y Sorensen, G. (2003). Employed adolescents and beliefs about self-efficacy to avoid smoking. Addictive Behaviors, 28, 613- 626.

Fishbein, M. (1990). Factores que influyen en la intención de estudiantes en decir a sus parejas que utilicen condón. Revista de Psicología Social y Personalidad, 6, 1-16.

Fishbein, M. (2000). The role of theory in HIV prevention. Aids Care, 12, 273- 278. 
Flay, B. R. y Petraitis, J. (1994). The theory of triadic influence: A new theory of health behavior with implications for preventive interventions. Advances in Medical Sociology, 4, 19- 44.

Gwaltney, C., Shiffman, S., Norman, G., Paty, Kassel, J., Gnys, M., Hickcox, M. y Balanbis, M. (2001). Does smoking abstinence self efficacy vary across situations? Identifying context- specificity with the relapse situation efficacy questionnaire. Journal of Consulting and Clinical Psychology, 66, 516- 527.

Landrum, S. K., Diamond, P. M., Dolan, M. P., Pallonen, U., Ford, K. H. y McAlister, A. L. (2007). Smoking- related self-efficacy, beliefs, and intention: Assessing factorial validity and structural relationships in 9th-12th grade current smokers. Addictive Behaviors, 32, 1863-1876.

Martin, G., Wilkinson, G. A. y Poulos, C. X. (1995). The drug avoidance self-efficacy scale. Journal of Substance Abuse, 7, 151-163.
Pérez G. A., Bermúdez, M. J. y Sanjuán S. P. (2000). Escala de autoeficacia general: datos psicométricos de la adaptación para población española Psicothema, 12 (Supl. 2), 509-513.

Prochaska, J. 0. y DiClemente, C. C. (1983). Stages and processes of self-change of smoking: Toward an integrative model of change. Journal of Consulting and Clinical Psychology, 51, 390-395.

Ortiz, A., Soriano, A., Galván, J. y Meza, D. (2005). Tendencias y uso de cocaina en adolescentes y jóvenes de la ciudad de México. Sistema de reporte de información en drogas. Salud Mental, 28, 91- 97.

Schonfield, P., Pattison, P. E., Hill, D. J. y Borland, R. (2003).Youth culture and smoking: Integrating social group processes and individual cognitive processes in a model of health-related behaviors. Journal of Health Psychology, 8, 291- 306. 